\title{
Quantum Teleportation of Optical Quantum Gates
}

\author{
Stephen D. Bartlett ${ }^{1}$ and William J. Munro ${ }^{2}$ \\ ${ }^{1}$ Department of Physics, Macquarie University, Sydney, New South Wales 2109, Australia \\ ${ }^{2}$ Hewlett-Packard Laboratories, Filton Road, Stoke Gifford, Bristol BS34 8QZ, United Kingdom
}

(Dated: 17 March 2003)

\begin{abstract}
We show that a universal set of gates for quantum computation with optics can be quantum teleported through the use of EPR entangled states, homodyne detection, and linear optics and squeezing operations conditioned on measurement outcomes. This scheme may be used for fault-tolerant quantum computation in any optical scheme (qubit or continuous variable). The teleportation of nondeterministic nonlinear gates employed in linear optics quantum computation is discussed.
\end{abstract}

PACS numbers: 03.67.Lx, 02.20.-a, 42.50.-p

Quantum computation (QC) may allow certain problems to be solved more efficiently than is possible on any classical machine [1] and optical realizations are particularly appealing because of the advanced techniques of quantum optics for state preparation, manipulation and measurement. However, many challenges still remain for fault-tolerant implementations of optical QC. In proposals for QC using optics [2, 3, 4], it is necessary to invoke some form of an optical nonlinear transformation, and unlike linear optics these nonlinear transformations either suffer from large losses (decoherence) or employ nondeterministic gates that fail a large fraction of the time.

The remarkable results by Gottesman and Chuang [5] show that quantum teleportation [6] can be used as a universal quantum primitive. In essense, quantum teleportation allows for the fault-tolerant implementation of "difficult" quantum gates that would otherwise corrupt the fragile information of a quantum state. The linear optics quantum computation (LOQC) scheme [2] relies on the quantum teleportation of nondeterministic nonlinear gates in the instances that they succeed in order to perform fault-tolerant QC. However, to overcome the difficulty in performing Bell-state measurements in this optical encoding using only linear optics [7], the LOQC scheme employs a near-deterministic quantum teleportation that places severe demands on the photodetectors employed for the measurements. The requirements to perform fault-tolerant quantum teleportation in this scheme greatly exceed current technologies.

Fortunately, there exists an alternative approach for quantum teleportation in optical systems: continuousvariable quantum teleportation (CVQT) [8, 9]. The measurements involved in this scheme are the highly developed and efficient techniques of homodyne detection [10]. Also, experimental CVQT can be performed unconditionally $\lfloor$, and does not suffer from the difficulties associated with the Bell-state measurements of qubit quantum teleportation [11]. It is of great interest, then, to determine what optical quantum gates can be teleported using CVQT (even in qubit schemes), and if such gates can be used to implement fault-tolerant optical QC.
In this Letter, we show that CVQT can easily teleport quantum gates that employ linear optics and squeezing operations. Also, by allowing squeezing operations conditioned on the results of measurements, we show that one can teleport optical nonlinear gates generated by some Hamiltonians that are cubic polynomial in the canonical coordinates. We prove that the gates which can be teleported in this fashion form a universal set of gates for $\mathrm{QC}$, and that this scheme allows for the fault-tolerant implementation of QC in any optical scheme employing qubits, qudits or CV encodings, provided one can perform fault-tolerant linear optics and squeezing. We analyze "noisy" teleportation employing finitely-squeezed states and imperfect detectors, and the resulting effect on the teleported gates. We conclude by discussing the challenges involved in using CVQT to teleport the nondeterministic gates in LOQC.

The transformations describing linear optics and squeezing possess a natural group structure which will be exploited for their quantum teleportation. Thus, we begin by reviewing the Pauli and Clifford groups for CV quantum computation [4, 12]. The standard Pauli group $\mathcal{C}_{1}$ on $n$ coupled oscillator systems is the Heisenberg-Weyl group, which consists of phase-space displacement operators for the $n$ oscillators. It is the group of "linear optics," which can be implemented by mixing states with strong coherent fields at a beamsplitter [13]. The algebra that generates this group is spanned by the $2 n$ canonical operators $\hat{q}_{i}, \hat{p}_{i}, i=1, \ldots, n$, along with the identity operator $\hat{I}$, satisfying the commutation relations $\left[\hat{q}_{i}, \hat{p}_{j}\right]=\mathrm{i} \hbar \delta_{i j} \hat{I}$. (In the following, we set $\hbar=1$.) For a single oscillator, the Pauli group consists of operators of the form $R(q, p)=\exp (-\mathrm{i}(q \hat{p}-p \hat{q}))$, with $q, p \in \mathbb{R}$. The Pauli operators for one system can be used to construct a set of Pauli operators $\left\{R_{i}\left(q_{i}, p_{i}\right) ; i=1, \ldots, n\right\}$ for $n$ systems (where each operator labeled by $i$ acts as the identity on all other systems $j \neq i$ ). This set generates the Pauli group $\mathcal{C}_{1}$. The Clifford group $\mathcal{C}_{2}$ is the group of transformations acting by conjugation that preserves the Pauli group $\mathcal{C}_{1}$; i.e., a gate $U$ is in the Clifford group if $U R U^{-1} \in \mathcal{C}_{1}$ for every $R \in \mathcal{C}_{1}$. The Clifford group $\mathcal{C}_{2}$ for continuous variables is easily shown [12] to 
be the (semidirect) product of the Pauli group and the linear symplectic group of all one-mode and two-mode squeezing transformations.

Transformations in the Clifford group do not form a universal set of gates for optical QC. However, Clifford group transformations together with any higher-order nonlinear transformation acting on a single mode form a universal set of gates 14]. Because arbitrary nonlinear gates can be constructed with such a set, this result also applies to qubit-based optical realizations for any encoding of qubits 2, 3, 4]. In the following, we construct a scheme to use CVQT to teleport such a universal set of gates.

Quantum teleportation, although initially proposed for remote parties, can also be used in a quantum circuit. Consider a three mode optical system (interferometer). Let mode 1 be in an arbitrary pure state $|\psi\rangle$ (although this circuit works equivalently well for mixed states), and modes 2 and 3 be in the maximally entangled EinsteinPodolsky-Rosen (EPR) state $|\mathrm{EPR}\rangle_{23}=\int|q\rangle_{2}|q\rangle_{3} \mathrm{~d} q$, where $|q\rangle$ are position eigenstates defined by $\hat{q}|q\rangle=q|q\rangle$. Modes 1 and 2 are then subjected to joint projective measurements of the form

$$
\Pi_{q, p}=R_{1}(q, p)|\mathrm{EPR}\rangle_{12}\langle\mathrm{EPR}| R_{1}^{\dagger}(q, p),
$$

where $R_{1}(q, p)$ is the Pauli operator on mode 1 . The measurement yields two classical numbers, $q_{0}$ and $p_{0}$, which are used to condition a Pauli operation $R_{3}\left(q_{0}, p_{0}\right)$ on mode 3 . The result of this circuit is that mode 3 is left in the state $|\psi\rangle$, and we say that this state has been quantum teleported to mode 3. A circuit diagram for quantum teleportation is given in the shaded box of Fig. 11 Repeating this circuit on many modes allows for the quantum teleportation of multi-mode states, preserving entanglement between modes.

We now consider how CVQT can be used to teleport a quantum gate. Consider an arbitrary transformation $S$ in the single mode $(n=1)$ Clifford group, and suppose we wish to implement this gate on an arbitrary single mode state $|\psi\rangle$. In the following, we show that it is possible to implement $S$ on one mode of an EPR state, and then employ a modified CVQT circuit to "teleport" this gate onto the desired state $|\psi\rangle$.

We use the property that, if $U$ is in the single mode Clifford group, then

$$
U R\left(q_{0}, p_{0}\right)=\left(U R\left(q_{0}, p_{0}\right) U^{-1}\right) U=R^{\prime}\left(q_{0}, p_{0}\right) U,
$$

and $R^{\prime}\left(q_{0}, p_{0}\right)=U R\left(q_{0}, p_{0}\right) U^{-1}$ is an element of the Pauli group due to the definition of the Clifford group, determined by the gate $U$. Thus, the desired Clifford gate $U$ can be quantum teleported onto the state $|\psi\rangle$ simply by implementing $U$ on one mode of the EPR pair and by appropriately altering the conditional displacement of the quantum teleportation. Using $n$ single-mode quantum teleportation circuits, it is then possible to teleport

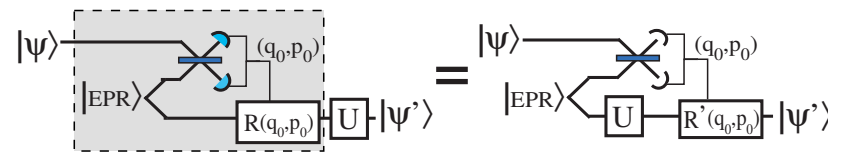

FIG. 1: Quantum teleportation of a gate $U$. The shaded box contains the CVQT circuit. The process of first teleporting the state then implementing $U$ (resulting is the state $\left|\psi^{\prime}\right\rangle=$ $U|\psi\rangle$ ) is equivalent to acting on one mode of the EPR state with $U$, followed by a modified quantum teleportation.

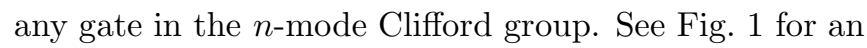
illustration of how this gate teleportation is performed.

In addition, this technique can be used to teleport more general quantum gates than those in the Clifford group. Consider the set of gates defined by $\mathcal{C}_{3}=\left\{U \mid U \mathcal{C}_{1} U^{-1} \subseteq\right.$ $\left.\mathcal{C}_{2}\right\}$. This set includes all gates that, when conjugating any Pauli group operation, yield a Clifford group operation. Note that $\mathcal{C}_{3}$ is not a group (it is not closed under composition) and contains nonlinear transformations. One example of a nonlinear gate in $\mathcal{C}_{3}$ is the cubic phase gate $V(\gamma)=\exp \left(\mathrm{i} \gamma \hat{q}^{3}\right)$, with $\gamma \in \mathbb{R}$, which Gottesman et al [4] have shown how to implement using Clifford group transformations and homodyne and photon counting measurements. (For details, see [15].) An example of a multimode gate in $\mathcal{C}_{3}$ is the controlled phase gate $C P_{i j}=\exp \left(\frac{1}{2} \mathrm{i} \hat{q}_{i} \hat{q}_{j}^{2}\right)$ acting on two modes $i$ and $j$. In comparison to the $\mathrm{CV}$ phase gate [12], this gate performs a phase gate operation on mode $j$, the magnitude of which depends on the state of mode $i$. It is a controlled Clifford group transformation; others can be defined similarly.

We now define a quantum teleportation circuit to teleport a gate $U \in \mathcal{C}_{3}$. Commuting this gate through the conditional operations gives

$$
U R\left(q_{0}, p_{0}\right)=\left(U R\left(q_{0}, p_{0}\right) U^{-1}\right) U=R_{2}^{\prime}\left(q_{0}, p_{0}\right) U,
$$

where, by the definition of $\mathcal{C}_{3}, R_{2}^{\prime}\left(q_{0}, p_{0}\right)$ is an element of the Clifford group. Thus, any gate in $\mathcal{C}_{3}$ can be quantum teleported using Clifford group transformations conditioned on measurement outcomes; i.e., conditional phasespace displacements and squeezing operations.

Consider, as an example, the CVQT of the cubic phase gate $V(\gamma)$. Commuting this gate back through the Pauli operator gives

$$
\begin{aligned}
R_{2}^{\prime}\left(q_{0}, p_{0} ; \gamma\right) & =V(\gamma) R\left(q_{0}, p_{0}\right) V(\gamma)^{-1} \\
& =\exp \left(-\mathrm{i}\left(q_{0} \hat{p}-p_{0} \hat{q}+3 \gamma q_{0} \hat{q}^{2}\right)\right)
\end{aligned}
$$

This operation, generated by an inhomogeneous quadratic polynomial in $\hat{q}$ and $\hat{p}$, lies in the Clifford group and can be implemented using a combination of conditional phase-space displacements and one-mode squeezing. Thus, to teleport $V(\gamma)$, this gate is performed on one mode of an EPR state, followed by a modified teleportation scheme with a conditional operation $R_{2}^{\prime}\left(q_{0}, p_{0} ; \gamma\right)$; the result is that a state $|\psi\rangle$ is quantum teleported into the transformed state $V(\gamma)|\psi\rangle$. 
The cubic phase gate $V(\gamma)$, being a higher-order nonlinear gate on a single mode, can be combined with Clifford group gates of $n$ modes to form a universal set of gates for QC on $n$ modes [14, and a scheme exists to implement this cubic phase gate [4]. Thus, with CVQT it is possible to teleport a universal and realizable set of gates. If Clifford group transformations and CVQT can be implemented fault-tolerantly, then it is possible to use this scheme to implement a fault-tolerant cubic phase gate (or other nonlinear gate in $\mathcal{C}_{3}$ ) using a gate that is not fault-tolerant. Thus, any nonlinear transformations can be moved "off-line" [4, 5]; although these transformations must still be performed in the quantum teleportation circuit, they can be made to act on EPR ancilla states non-deterministically rather than on the fragile encoded states.

Because most optical quantum information schemes employ the Kerr effect (generated by a Hamiltonian of the form $\left.\left(\hat{a}^{\dagger}\right)^{2} \hat{a}^{2}\right)$ as the nonlinear transformation outside of the Clifford group, it is of interest to consider how such a transformation can be implemented using the above universal set of gates. Using the relation $e^{\mathrm{i} A t} e^{\mathrm{i} B t} e^{-\mathrm{i} A t} e^{-\mathrm{i} B t}=e^{\mathrm{i}[A, B] t^{2}}+\mathcal{O}\left(t^{3}\right)$, a combination of cubic phase gates and Clifford gates can be used to simulate the Kerr nonlinearity to any degree of accuracy. We also note that it is possible to iteratively define more higher-order sets of gates $\mathcal{C}_{k}=\left\{U \mid U \mathcal{C}_{1} U^{-1} \subseteq \mathcal{C}_{k-1}\right\}$ that can be implemented fault-tolerantly if gates in $\mathcal{C}_{k-1}$ can be implemented fault-tolerantly. The Kerr nonlinearity is related to transformations in the set $\mathcal{C}_{4}$.

We now consider the effects of realistic noise and errors that may occur in this scheme, and show that quantum error correcting codes (QECCs) may allow for faulttolerant quantum computation. Clifford group transformations describing linear optics and squeezing will in general contain errors such as imprecise transformations (e.g., imperfect beamsplitter reflectivities) or linear coupling to the environment such as amplitude damping. Due to the linear nature of these couplings, such errors are described by "small" Clifford group transformations; it is unlikely that a large nonlinear error will occur in an optical system. For example, errors in the implementation of a displacement operation may be described by small displacement errors $R(\delta q, \delta p)$ with, say, a Gaussian distribution; see below. Thus, we represent such errors in Clifford group transformations by distributions of linear transformations $R(\delta q, \delta p)$, where $\delta q^{2}+\delta p^{2}<S^{2}$ for some maximum error distance $S$ in phase space.

To describe errors in CVQT, we must take into account finite squeezing and imperfect detection. In experiment 9], the EPR states are approximated by two-mode squeezed vacua 16

$$
|\eta\rangle_{23}=\sqrt{1-\eta^{2}} \sum_{n=0}^{\infty} \eta^{n}|n\rangle_{2}|n\rangle_{3}
$$

with $|n\rangle$ the $n$-boson Fock state and $0<\eta \leq 1$. In the limit $\eta \rightarrow 1$, this state becomes the maximally entangled EPR state $|\mathrm{EPR}\rangle_{23}$. The projective measurements of Eq. (11) are implemented via homodyne detection, and the displacement operation $R_{3}\left(q_{0}, p_{0}\right)$ is performed by mixing the field with a local oscillator at a beamsplitter; see 8, 9] for details. Finite squeezing $\eta<1$ and imperfect homodyne efficiency $\nu<1$ can characterised by a Gaussian noise term with variance $\sigma=\exp \left(-2 \tanh ^{-1} \eta\right)+\left(1-\nu^{2}\right) / \nu^{2}$, defined such that $\sigma=1 / 2$ is the level of vacuum noise [17]. For demonstrated CVQT with fidelity $F>0.5$, we require $\sigma<1$; for fault-tolerant teleportation of quantum gates, $\sigma$ must be significantly smaller unless appropriate quantum error correction can be applied (see below).

Imperfect quantum teleportation can be described by a transfer superoperator $\mathcal{E}_{\sigma}$ defined on a state $\rho$ as

$$
\mathcal{E}_{\sigma}(\rho)=\int \frac{\mathrm{d} q \mathrm{~d} p}{\pi \sigma} \exp \left(-\frac{q^{2}+p^{2}}{\sigma}\right) R(q, p) \rho R^{\dagger}(q, p) .
$$

The transfer superoperator has the effect of convoluting the state $\rho$ with a Gaussian of standard deviation $\sigma$. Let $\mathcal{E}_{U}$ be the superoperator corresponding to a unitary transformation $U$, defined on a density matrix $\rho$ to be $\mathcal{E}_{U}(\rho)=U \rho U^{-1}$. Using noisy quantum teleportation to teleport the gate $U$, the resulting transformation is described by the superoperator $\mathcal{E}_{U} \circ \mathcal{E}_{\sigma}$. If $\mathcal{E}_{U}^{\prime}$ describes a gate implementing the unitary transformation $U$ with small linear noise, the superoperator $\mathcal{E}_{U}^{\prime} \circ \mathcal{E}_{\sigma}$ describes a teleported gate with an additional Gaussian noise due to the teleportation.

Thus, noisy quantum teleportation of gates is described by a convolution of the (possibly noisy) gate with a Gaussian noise process; the corresponding errors can be viewed as random displacements $R(\delta q, \delta p)$ with $\delta q^{2}+\delta p^{2} \sim \sigma$. The effect of this Gaussian noise and the existence of QECCs to correct this noise depends critically on the specific encoding used. Single photon encodings 2] and superpositions of coherent states [3] are extremely fragile to Gaussian noise. The encoding of Gottesman et al [4], however, is specifically designed to protect against such Gaussian noise because small displacements in phase space can be detected and corrected. Briefly, this encoding employs superpositions of highly squeezed states, for which small displacement errors $R(\delta q, \delta p)$ can be detected and corrected using only Clifford group transformations and homodyne detection. As long as the displacement errors in the Clifford group transformations and the CVQT are sufficiently small compared to the length scale (in phase space) of the encoding, quantum error correction can be applied frequently enough to keep these errors small and contained; see 4] for details. In addition, these codes can be concatenated with the QECCs of 18], which protect against large displacement errors on a single mode. Thus, our proposal for the quantum teleportation of nonlinear gates 
can be made fault-tolerant if encodings are used that are protected against Gaussian noise and the QECCs use fault-tolerant Clifford group operations (i.e., operations with small errors in the above sense). Creating the encoded states of [4] is extremely challenging because it requires coherent superpositions of highly squeezed states. These Gaussian noise processes, however, are indicative of optical systems, and the investigation of "Gaussian protected" encodings that require only Clifford group operations is essential for robust quantum information processing using optics.

Because CVQT can teleport any state of an optical mode, we can consider the use of CVQT in any optical quantum information process, even qubit-based schemes. One scheme 3] encoding qubits as coherent states could employ CVQT to implement the "difficult" Hadamard transformations requiring a Kerr nonlinearity in a fault-tolerant way. In LOQC, nondeterministic gates are used to implement nonlinear transformations on arbitrary states with up to two photons. Qubit-based quantum teleportation is employed to implement these gates fault-tolerantly, but the near-deterministic quantum teleportation of LOQC places strong demands on photodetectors and requires highly entangled multimode states. To instead employ CVQT, the problem arises that any gate to be teleported must be made to act on one mode of an EPR state. The probability of a successful gate operation may be affected by the photon number cutoff used for these states (the nonlinear sign gate being one such example). For high-fidelity quantum teleportation, one must employ highly squeezed states that have a corresponding high photon number cutoff (see Eq. (2)); thus, any effective teleportable gate must be designed to operate on such high-photon states. However, the challenges to design such a non-deterministic gate that can be teleported using this scheme may be less significant than the difficulties involved in the near-deterministic teleportation of LOQC.

In conclusion, we have shown that fault-tolerant implementations of Clifford group transformations and CVQT is sufficient to perform fault-tolerant QC in any optical scheme: qubits, qudits or CV. A nonlinear transformation such as the cubic phase gate (possible using Clifford group transformations, homodyne detection, and photon counting) can be performed "off-line" on EPR states and used as a resource to perform universal QC. Such gates would allow the implementation of difficult but critical nonlinear transformations such as the Kerr effect in a fault-tolerant way. The definition of an infinite series of gates $\mathcal{C}_{k}$ highlights which gates can be teleported in a straightforward manner. In particular, the cubic phase gate (rather than a Kerr nonlinearity) is identified as the most direct nonlinear operation that would achieve a universal set of gates. The simplicity and power of this gate clearly motivates its experimental demonstration and its use in future quantum information processing tasks. The use of CVQT in LOQC relies on the design of new nondeterministic nonlinear gates that can be teleported using this scheme. Finally, the Gaussian noise introduced through realistic CVQT can be corrected with suitable "Gaussian protected" encodings and Clifford group transformations, and the results presented here highlight the need for new, realistic quantum error correction schemes of this form.

This work was supported in part by the European Union through RAMBOQ and the QUIPROCONE Network, and by Macquarie University. We acknowledge helpful discussions with K. Nemoto, T. Ralph and B. C. Sanders.

[1] M. A. Nielsen and I. L. Chuang, Quantum Computation and Quantum Information, (Cambridge University Press, Cambridge, 2000).

[2] E. Knill et al, Nature (London) 409, 46 (2001).

[3] T. C. Ralph et al, quant-ph/0110115 (2001).

[4] D. Gottesman et al, Phys. Rev. A 64, 012310 (2001).

[5] D. Gottesman and I. L. Chuang, Nature (London) 402, 390 (1999).

[6] C. H. Bennett et al, Phys. Rev. Lett. 70, 1895 (1993).

[7] N. Lütkenhaus et al, Phys. Rev. A 59, 3295 (1999).

[8] S. L. Braunstein and H. J. Kimble, Phys. Rev. Lett. 80, 869 (1998).

[9] A. Furusawa et al, Science 282, 706 (1998).

[10] E. S. Polzik et al, Phys. Rev. Lett. 68, 3020 (1992).

[11] S. L. Braunstein and H. J. Kimble, Nature (London) 394, 840 (1998).

[12] S. D. Bartlett et al, Phys. Rev. Lett. 88, 097904 (2002).

[13] D. F. Walls and G. J. Milburn, Quantum Optics, (Springer, Berlin, 1994).

[14] S. Lloyd and S. L. Braunstein, Phys. Rev. Lett. 82, 1784 (1999).

[15] S. D. Bartlett and B. C. Sanders, Phys. Rev. A 65, 042304 (2002).

[16] S. J. van Enk, Phys. Rev. A 60, 5095 (1999).

[17] T. J. Johnson et al, Phys. Rev. A 66, 042326 (2002).

[18] S. Lloyd and J.-J. E. Slotine, Phys. Rev. Lett. 80, 4088 (1998); S. L. Braunstein, Phys. Rev. Lett. 80, 4084 (1998); S. L. Braunstein, Nature (London) 394, 47 (1998). 